\title{
Produção orgânica: predileção de consumo e potencialidades do seguimento em Fortaleza - CE
}

\section{Organic production: consumer preference and potential tracking in Fortaleza - CE}

\author{
Carmem Sara Pinheiro de Oliveira ${ }^{1}$, Allison Ferreira de Lima ${ }^{2}$, Ewerton Calixto da Silva ${ }^{3}$, Weibson Paz Pinheiro Andre ${ }^{4}$ \\ Thais Paz Pinheiro Andre
}

\begin{abstract}
Resumo: Este trabalho objetivou investigar o perfil de consumidores de produtos orgânicos, bem como sua forma de aquisição e quais as preferências que influenciam no momento de decidir sobre a compra. O presente estudo foi desenvolvido durante a VIII FECEAF - Feira Cearense da Agricultura Familiar - em Fortaleza, Ceará, durante julho de 2015, totalizando 100 sujeitos amostrais. Foram respondidas questões objetivas referentes ao perfil do consumidor, onde os indivíduos do sexo feminino (51\%), à faixa etária entre 21 a $30(39 \%)$ e o ensino médio (48\%) desse público obtiveram maior representatividade. Observouse ainda o pouco conhecimento acerca dos produtos orgânicos entre os entrevistados (61\%), tendo a televisão como principal fonte de informação a respeito do tema (34\%). No que diz respeito aos hábitos de consumo e a forma de aquisição desse tipo de produto, $54 \%$ dos respondentes o escolhem por apresentar uma melhor qualidade quando comparados aos convencionais, $43 \%$ o consomem todos os dias e $41 \%$ costumam adquiri-los em supermercados. Já o hábito familiar de consumo obteve um percentual expressivo (87\%), em que todos os integrantes da família fazem uso do consumo de produtos orgânicos. Quanto à atratividade, $53 \%$ relatam que a ausência de agrotóxicos tornam os produtos orgânicos mais atrativos. Entre os orgânicos mais consumidos, as frutas (53\%) e as hortaliças (29\%) obtiveram maior destaque. Nesse sentido concluímos com o trabalho que os hábitos alimentares das famílias tem se tornado cada vez mais saudáveis, dando espaço ao crescimento do comércio do produto orgânico, fortalecendo o mercado incentivando a produção.
\end{abstract}

Palavras-chaves: Agricultura agroecológica, Meio ambiente, Perfil socioeconômico, Sustentabilidade.

Abstract: This study aimed to investigate the profile of consumers of organic products as well as their way of acquisition and which preferences that influence when deciding on the purchase. This study was developed during the VIII FECEAF - Fair Cearense Family Agriculture - Fortaleza, Ceará, in July 2015, totaling 100 sample subjects. objective questions were answered for the consumer profile, where the females (51\%), the age group 21-30 (39\%) and high school (48\%) of that audience got more representative. There was still little knowledge about organic products among respondents (61\%), with television as the main source of information on the subject (34\%). With regard to consumer habits and how to purchase this type of product, $54 \%$ of respondents choose it to present a better quality when compared to conventional, $43 \%$ consume it every day and $41 \%$ usually buy them in supermarkets . Already familiar consumption habits obtained a significant percentage (87\%) in all family members making use of organic consumer products. As for attractiveness, 53\% report that the absence of pesticides makes them more attractive organic products. Among the most consumed organic, fruits (53\%) and vegetables (29\%) were most prominent. In this sense we completed the work that the eating habits of families has become increasingly healthy, making room for the growth of the organic product trade, strengthening the market by encouraging production.

Keywords: Agroecological agriculture, Environment, Health, Socioeconomic profile, Sustainability

\footnotetext{
*Autor para correspondência

Recebido para publicação em 28/08/2015; aprovado em 10/09/2015

${ }^{1}$ Mestranda em Aquicultura, Universidade Federal de Santa Catarina, Florianópolis; (48) 98527033, E-mail: sarinhac.s@hotmail.com.

${ }^{2}$ Graduando em Zootecnia, Universidade Federal Rural do Semi-Árido, E-mail: henresito@ hotmail.com

${ }^{3}$ Graduado em Ecologia, Universidade Federal do Rio Grande do Norte, E-mail: calixtoewerton@ @otmail.com

${ }^{4}$ Mestrando em Ciências Veterinárias, Universidade Estadual do Ceará, E-mail: weibsonpaz@ hotmail.com

${ }^{5}$ Graduanda em Agronomia, Universidade Federal Rural do Semi-Árido, E-mail: thais-agronomia@ hotmail.com
} 


\section{INTRODUÇÃO}

Atualmente uma das palavras mais utilizadas para demonstrar que os recursos devem ser utilizados visando também às gerações futuras é a sustentabilidade (BRAGA, 2014). Neste contexto, surge a agricultura orgânica, também conhecida como agricultura agroecológica, que é um setor de grande crescimento mundial. Segundo Lombard et al. (2004), os produtos orgânicos possuem a ausência de fertilizantes químicos e agrotóxicos em seu cultivo, tornando-os assim mais atrativos.

Mediante normas do Ministério da Agricultura, Pecuária e Abastecimento, fundamentadas na Lei $\mathrm{N}^{\circ} 710.831$, de 23 de dezembro de 2003, o sistema de produção de agropecuária e industrial embasados em produtos orgânicos é entendido como aquele em que se adotam tecnologias que otimizem o uso de recursos naturais e socioeconômicos, respeitando a integridade cultural e tendo por objetivo a autosustentação, a maximização dos benefícios sociais, a minimização da dependência de energias não renováveis e a eliminação do emprego de agrotóxicos e outros insumos artificiais tóxicos, organismos geneticamente modificados ou radiações ionizantes em qualquer fase do processo de produção, armazenamento e de consumo.

As causas para o consumo de produtos orgânicos variam de acordo com o país, preço, produtos analisados e da cultura. Em alguns países como Alemanha, Austrália, Dinamarca, Inglaterra e Estados Unidos, o consumidor desses produtos leva primeiramente em consideração o quão saudável é aquele produto, em segundo lugar os aspectos pertinentes ao meio ambiente (DAROLT, 2001). Nos países em desenvolvimento um dos empecilhos que dificulta o crescimento desse tipo de cultura é o alto preço dos produtos quando comparados com os produtos convencionais (SILVA et al., 2008).

De acordo com Zamberlan, Büttenbender e Sparemberger (2006), a crescente demanda por produtos orgânicos está fortemente relacionada ao aumento da exigência dos consumidores, internos e externos, com a qualidade dos alimentos e com os impactos da agricultura sobre o meio ambiente. Essas profundas mudanças no setor agroalimentar alteram fortemente a produção e a oferta de alimentos.

Assim, toma-se como um novo lugar a nova consciência alimentar, que pressupõe que a escolha ética implica no conhecimento de como, onde e de que modo os alimentos são produzidos e comercializados. No caso dos produtos orgânicos, cujo crescimento e a expansão do mercado produtor e consumidor tornam-se expressivos, revela uma nova consciência que tem sido analisada seguindo, principalmente, a preocupação com o meio ambiente, com o bem-estar e como um modo de ação política (KRISCHKEL; TOMIELLO, 2009).

A crescente demanda por alimentos produzidos com menos agrotóxicos e menos agressivos ao meio ambiente é uma tendência mundial que se reflete também no Brasil. Tal procura tem como consequência a geração de novas oportunidades de negócio para os vários segmentos da agropecuária nacional (BUAINAIN; BATALHA, 2007).

Pereira e Torres Filho (2010), afirmam que as Feiras agroecológicas são espaços para comercialização de produtos orgânicos, sendo as mesmas consideradas como uma forma alternativa de proporcionar para população alimentos mais saudáveis, que optam e procuram por produtos frescos, de preferência diretamente do produtor rural.

Durante a realização dessas feiras, existe a participação em massa de famílias rurais, no qual a comercialização de seus produtos viabiliza a possibilidade de um contato direto com o cliente, mediando trocas de conhecimento e a estruturação de uma economia mais justa, podendo assim expandir a comercialização deste tipo de produto.

Todavia, mesmo crescendo de forma exponencial nas ultimas décadas a produção de produtos orgânicos ainda representa cerca de apenas $15 \%$ dos produtos comercializados em todo o Brasil, decorrente do seu tipo de cultivo e produção em menor escala. Para Silva e Rocha (2003) os produtos convencionais, por sua vez, detêm cerca de $85 \%$ da produção, sendo muito difundido na exportação. O estado do Ceará possui cerca de $10 \%$ de consumidores de produtos orgânicos, o que ainda é considerado pouco, entretanto a nível nacional é um dos estados que mais consomem tal produto.

Considerando o cenário sócio-econômico-ambiental, este trabalho objetivou investigar o perfil de consumidores de produtos orgânicos, bem como sua forma de aquisição e quais as preferências que influenciam no momento de decidir sobre a compra na capital cearense.

\section{MATERIAL E MÉTODOS}

A coleta de dados se deu a partir da visitação à VIII FECEAF - Feira Cearense da Agricultura Familiar, considerada a maior feira de origem da agricultura familiar no estado do Ceará, no qual objetiva a divulgação, exposição $e$ comercialização de produtos de origem agrícola. A FECEAF foi realizada no município de Fortaleza, Ceará, durante o período de 3 a 5 de julho do ano de 2015. Foram entrevistados 100 consumidores de ambos os sexos, com idades variadas, não sendo realizada a identificação dos mesmos.

O questionário foi embasado nesses estudos (ZAMBERLAN et al., 2006; CUENCA et al., 2007). Esta pesquisa é de caráter quali-quantitativa e de natureza exploratória, oriunda da aplicação de questionários aplicados junto a consumidores de produtos orgânicos. Como não se tem uma estimativa concreta do número de pessoas que consomem estes produtos, utilizou-se uma amostra aleatória, satisfazendo as necessidades do estudo.

Os questionários foram elaborados com questões de múltipla escolha relacionadas ao perfil socioeconômico dos consumidores orgânicos, além de características de preferência e hábitos, permitindo assim que os entrevistados pudessem responder todas as perguntas, visando uma melhor interpretação dos dados que foram obtidos na pesquisa.

Após a aplicação dos questionários, os dados obtidos foram tabulados e analisados através da estatística descritiva por meio do percentual da frequência das respostas encontradas.

\section{RESULTADOS E DISCUSSÃO}

De acordo com dados obtidos a maioria dos entrevistados é do sexo feminino representado por $51 \%$, mas um percentual não significativamente diferente é do sexo masculino que ficou com $49 \%$. Essa constatação reforça a tese levantada por Silva et al. (2008) em que a maioria dos 
consumidores de produtos orgânicos pertencem ao grupo do sexo feminino.

Na tabela 1 é possível perceber que o maior percentual de consumidores, levando em consideração o público participante da pesquisa, foi com os que possuem idade entre 21 a 30 anos com $39 \%$, seguido por: idade igual ou inferior a 20 anos com 30\%; de 31 a 40 anos com 15\%; de 41 a 50 anos com 6\%; e de 51 a 60 anos, bem como idade igual ou superior a 60 anos com 5\% cada. Os indivíduos com idades variando entre 21 a 30 anos podem ser o público alvo para consumir produtos orgânicos, pois os jovens, ao contrário das crianças e indivíduos mais velhos, levam em consideração aspectos mais complexos ao ingerir determinado alimento e a capacidade de adicionar novos alimentos na dieta alimentar variam pouco da adolescência até a idade adulta (VIANA et al., 2008).

Ainda na tabela abaixo, quanto ao grau de escolaridade, $5 \%$ alegaram ser analfabetos, $33 \%$ disseram ter concluído o ensino fundamental, enquanto que a maioria com $48 \%$ estudaram até o ensino médio, já $8 \%$ possuem o ensino superior como maior nível de formação e sujeitos com pósgraduação foram representados aqui por $6 \%$, essas dados são corroborados por Ferreira e Marinho (2013), no qual observaram que $47,42 \%$ das pessoas entrevistadas possuíam o nível médio completo.

Tabela 1 - Distribuição por faixa etária e por nível de escolaridade dos entrevistados.

\begin{tabular}{cccc}
\hline \multicolumn{2}{c}{ Faixa etária } & \multicolumn{2}{c}{ Nível de escolaridade } \\
\hline Idade & $\mathbf{\%}$ & Maior nível de formação & \% \\
\hline$\leq 20$ & 30,00 & Analfabeto & 5,00 \\
De 21 a 30 & 39,00 & Ensino Fundamental & 33,00 \\
De 31 a 40 & 15,00 & Ensino Médio & 48,00 \\
De 41 a 50 & 6,00 & Ensino Superior & 8,00 \\
De 51 a 60 & 5,00 & Pós-graduação & 6,00 \\
$\geq 61$ & 5,00 & ----- & --- \\
\hline Total & $\mathbf{1 0 0}$ & Total & $\mathbf{1 0 0}$ \\
\hline
\end{tabular}

$\mathrm{Na}$ literatura consultada a diferença entre o consumo dos produtos orgânicos entre as classes sociais é grande. A porcentagem de pessoas da classe A que adquirem orgânicos é mais que o dobro da porcentagem de pessoas que têm este hábito da classe $\mathrm{B}$ e $\mathrm{C}$ e quase o triplo da classe D (BRAGA, 2014). O que mostra que além da qualidade e segurança alimentar, o valor pago pelo produto ainda é um fator limitante do seu consumo, tendo em vista o seu alto preço no mercado.

Os dados também apontaram que a maioria dos entrevistados $61 \%$ possuíam pouco conhecimento acerca dos produtos orgânicos, como por exemplo: "Para que servem esses produtos?", “Como são produzidos?", "Qual a diferença entre produtos orgânicos e produtos convencionais?"; e assim por diante. Logo depois $32 \%$ dos entrevistados demonstraram possuir um grande conhecimento sobre esses produtos e $7 \%$ não conhecem nada sobre esses produtos, resultados similares foram encontrados por Cuenca et al. (2007).

Dos cem entrevistados, 34\% disseram que o conhecimento adquirido acerca dos produtos orgânicos se deu por meio da televisão seguida pelas revistas com $16 \%$, internet com $12 \%$, palestras com $10 \%$, jornais $9 \%$, rádio com $8 \%$, os demais $11 \%$ afirmam não possuir conhecimentos adequados acerca desses produtos. Em um estudo na Bahia, a televisão (35\%) e as revistas $(15 \%)$ também foram os meios de comunicação mais citados pelos consumidores de produtos orgânicos. A televisão se destaca como uma mídia digital importante como fonte de disseminação de conhecimento mundial nas ultimas décadas. Os meios de comunicação devem ser utilizados para disseminar o conhecimento dos benefícios de consumir produtos orgânicos e apoiar a comercialização desses produtos (CASEMIRO; TRAVISAN, 2009).

Em relação ao que os consumidores orgânicos levam em consideração para a compra destes produtos, mais da metade dos entrevistados (54\%) escolhem esses produtos por apresentarem uma melhor qualidade quando comparados com os produtos convencionais, ao passo que (45\%) dos entrevistados elegeram a segurança alimentar como o item mais importante para a escolha do produto, apenas (1\%) afirmou possuir dieta específica. Esse um estudo no Rio Grande do Norte, $80 \%$ dos entrevistados indicaram que confiam que os produtos orgânicos sejam seguros para saúde humana (CUENCA et al., 2007). Os alimentos orgânicos representam alimentação mais saudável, preservação do meio ambiente e geração de emprego e renda no campo e gerando emprego no campo (CASEMIRO; TRAVIZAN, 2009).Com os dados adquiridos foi possível observar com qual frequência os consumidores orgânicos consomem esses produtos. A grande maioria dos entrevistados (43\%) consomem os produtos todos os dias, enquanto que (32\%) dos entrevistados ingerem os produtos semanalmente, já (15\%) quinzenalmente e $(10 \%)$ mensalmente. Quando questionados acerca da sua forma de aquisição destes produtos os consumidores pontuaram os seguintes estabelecimentos de venda: supermercados $(41 \%)$, sob encomenda (4\%), feiras livres $(31 \%)$ e direto com o produtor $(24 \%)$. Buainain e Batalha (2007), afirmam que os consumidores de produtos orgânicos são formados em sua grande maioria por frequentadores de feiras verdes de produtos orgânicos e por consumidores das grandes redes de supermercados, corroborando os dados acima.

No que concerne o hábito do consumo familiar de produtos orgânicos na atualidade, pudemos observar que mediante um percentual com um alto nível de significância afirmam que todos os integrantes da família fazem uso do consumo (87\%), o que mostra que muitas vezes o fator "família" é a influencia, já apenas o participante da pesquisa em sua residência consome produtos orgânicos (10\%), apenas os adultos $(3 \%)$ e apenas as crianças $(0 \%)$.

Segundo Hoppe et al. (2012), os padrões de consumo de alimentos estão mudando rapidamente hoje em dia, como resultado de questões de desenvolvimento e sustentabilidade, considerações com relação ao seu aspecto nutricional e também questões relacionadas à saúde. O que pôde ser evidenciado na pergunta sobre o que é mais atrativo neste tipo 
de produto, no qual a ausência de agrotóxicos foi representado pela maior porcentagem como mostra a figura 1 .

No que diz respeito à preferência dos diferentes tipos de produtos orgânicos a grande maioria opta pelo que apresenta maior diversidade e custo menos elevado, além da facilidade de aquisição, como é o caso das frutas e hortaliças (figura 2).

Figura 1. Porcentagem representando o que é mais atrativo em produtos orgânicos.

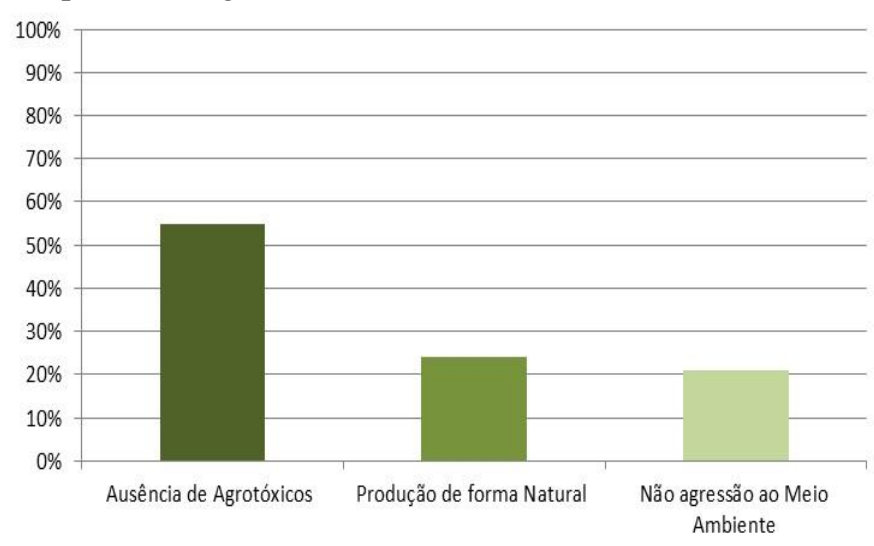

Figura 2. Percentual de tipos de produtos preteridos por consumidores de orgânicos.

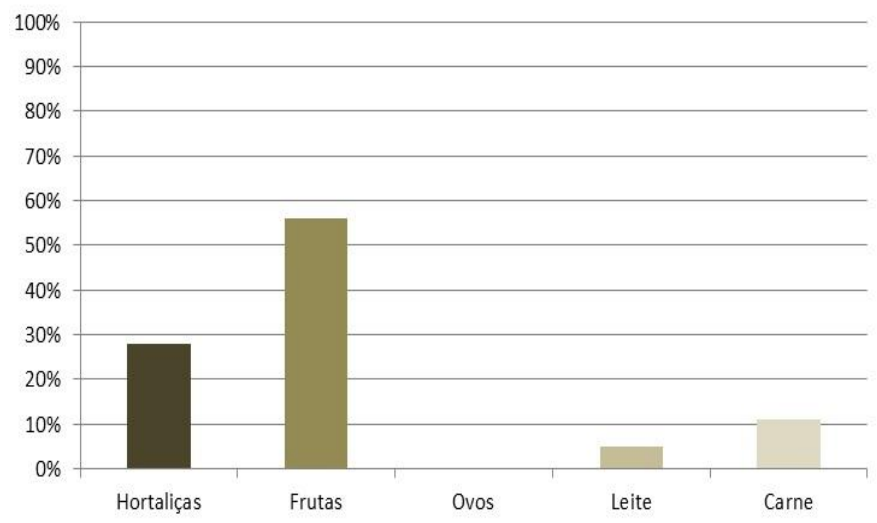

Quanto aos produtos mais consumidos, seja de origem animal ou vegetal, encontramos as seguintes porcentagens: Banana 16\%, Tomate 15\%, Maçã 15\%, Mamão 9\%, Laranja $9 \%$, Coentro 9\%, Alface 7\%, Carne bovina 5\%, Leite de vaca $4 \%$, Carne de frango $3 \%$, Acerola 2\%, Melancia $1 \%$, Gergelim 1\%, Feijão 1\%, Carne suína $1 \%$ e Abacaxi $1 \%$, confirmando os dados mostrados anteriormente no qual apresentam maior preferência, e por consequência, maior consumo para as frutas.

Sendo assim, produtos produzidos a partir de cultivos que não agridam o meio e a saúde humana são potencialmente preteridos. A aceitabilidade dos produtos orgânicos tem sido evidenciada em pesquisas relacionadas a essa importante temática.

\section{CONCLUSÕES}

Os hábitos alimentares das famílias têm se tornado cada vez mais saudáveis, dando espaço para o crescimento do comércio de produtos orgânicos. A alta demanda desses produtos, fortalece o mercado incentivando a produção
A segurança alimentar e qualidade nutricional ainda são os fatores mais marcantes no tocante da escolha do consumo orgânico, uma vez que a população está cada vez mais preocupada com a saúde. A preferência por frutas e hortaliças é notória, almejando sempre a qualidade de vida.

\section{REFERÊNCIAS BIBLIOGRÁFICAS}

BRAGA, M. O comportamento de compra de produtos orgânicos: uma reflexão sobre o perfil dos consumidores através da comparação entre faixas etárias e níveis de renda familiar - Uma abordagem estatística. Sumaré revista acadêmica eletrônica. 2014. Disponível em: <http://www.sumare.edu.br/Arquivos/1/raes/8_9/compor tamento-compra-organicos.pdf $>$. Acesso em: jul/2015.

BRASIL. Ministério da Agricultura Pecuária e Abastecimento - Lei No 710.831, de 23 de dezembro de 2003. Dispõe sobre a agricultura orgânica e da outras providências. Disponivel em: http://www.planalto.gov.br/CCIVIL_03/leis/2003/L10.8 31.htm. Acesso em: jun/2015.

BUAINAIN, A. M.; BATALHA, M. O. Agronegócios: cadeia produtiva de produtos orgânicos. Brasília, 2007. Disponível em: http://www.ibraf.org.br/x_files/Documentos/Cadeia_Pro dutiva_de_Produtos_Org\%C3\%A2nicos_S\%C3\%A9rie_ Agroneg\%C3\%B3cios_MAPA.pdf. Acesso em: jun/2015.

CASEMIRO, A. D.; TREVIZAN, S. D. P. Alimentos Orgânicos: Desafios para o Domínio Público de um Conceito. $2^{\text {nd }}$ International Workshop |Advances in Cleaner Production. Disponivel em: $<$ http://www.advancesincleanerproduction.net/secon $\mathrm{d} / \mathrm{fil}$ s/sessoes/6a/1/A.\%20D.\%20Casemiro\%20\%20Resumo $\% 20$ Exp.pdf>. Acesso em; mar/2016.

CUENCA, M. A. G.; MOREIRA, M. A. B.; NUNES, M. U. C.; MATA, S. S. da; GUEDES, C.G.M.; BARRETO, M. de F. P.; LÓPEZ, V.R.M. ; PAZ, F. das C. A.; SILVA, J.R. da; TORRES, J.F.Caracterização das Bases de produção orgânica no Rio Grande do Norte. Aracaju:(Embrapa, 124), p.20 , 2007.

DAROLT, M. R. O Papel do Consumidor no Mercado de Produtos Orgânicos. Agroecologia Hoje, v.7, p.8-9, 2001.

FERREIRA, S.;MARINHO, J. A. Levantamento do conhecimento da população urbana de Inconfidentes/MG sobre orgânicos. Revista Agrogeoambiental, v.5, p.3948, 2013.

HOPPE, A.; BARCELLOS, M. D.; VIEIRA, L. M.; MATOS, C. A. COMPORTAMENTO DO CONSUMIDOR DE PRODUTOS ORGÂNICOS: UMA APLICAÇÃO DA TEORIA DO COMPORTAMENTO PLANEJADO. 
Revista de Administração e Contabilidade da Unisinos, v.9,n.2, p.174-188, 2012.

KRISCHKEL, P.J.; TOMIELLO, N. O comportamento de compra dos consumidores de alimentos orgânicos: um estudo exploratório. Cadernos de Pesquisa Interdisciplinar em Ciências Humanas, v.10, n.96, p.2743, 2009.

LOMBARDI, Marta Sambiase; MOORI, Roberto Giro; SATO, Geni Satiko. Um Estudo Exploratório dos Fatores Relevantes na Decisão de Compra de Produtos Orgânicos. Revista de Administração Mackenzie,, n. 1, p. $13-34,2004$.

PEREIRA, J. S.; TORRES FILHO, J. Uma visão preliminar da feira agroecológica da ACB no município do CratoCE. In: II Encontros Universitário da UFC Campus Cariri, Juazeiro do Norte - Ceará. p. 01-04, 2010.

SILVA, A. M. N.; ALBUQUERQUE, J. L.; FILHO, D. S. F.;NASCIMENTO, R. P. L.;SILVA, E. L. Uma análise do perfil dos consumidores de produtos orgânicos em Pernambuco: o caso da feira agroecológica Chico Mendes - Recife- PE. In: XLVI Congresso da Sociedade Brasilleira de Economia e Sociologia Rural, Rio Branco, 2008.

SILVA, C. L.; ROCHA, J. H. M. V. 2003. Portas abertas para os orgânicos. Disponível em: <http://www.agronline.com.br/artigos/artigo.php?id=24> . Acesso em: jun/2015.

VIANA, V., SANTOS, P. L. \& GUIMARÃES, M. J., COMPORTAMENTO E HÁBITOS ALIMENTARES EM CRIANÇAS E JOVENS: Uma revisão da literatura. psicologia, saúde \& doenças, v. 9, n.2, v. 209-231, 2008.

ZAMBERLAN, L.; BÜTTENBENDER, P. L.; SPAREMBERGER, A. O comportamento do consumidor de produtos orgânicos e seus impactos nas estratégias de marketing. In: ENCONTRO ANUAL DA ANPAD, Salvador, 2006. 